\title{
THE GAP PHENOMENON IN THE DIMENSION STUDY OF FINITE TYPE SYSTEMS
}

\author{
BORIS KRUGLIKOV
}

\begin{abstract}
In this paper several examples of gaps (lacunes) between dimensions of maximal and submaximal symmetric models are considered, which include investigation of number of independent linear and quadratic integrals of metrics and counting the symmetries of geometric structures and differential equations. A general result clarifying this effect in the case, when the structure is associated to a vector distribution, is proposed.
\end{abstract}

\section{INTRODUCTION}

Finite type systems in differential geometry and differential equations are systems whose solution spaces are finite-dimensional. They are encoded by integrable vector distributions on manifolds. Solutions of the latter are integral surfaces, which by Frobenius theorem can be found through integration of ODEs.

Several enumeration problems arise in this respect. The most important are counting of symmetries and counting of the first integrals. Indeed, these object allow to solve the differential equations effectively. The larger is their amount, the easier is the integration.

Notice that symmetries of differential equations can be of different types: internal, external, higher etc. Lie-Bäcklund type theorems in some cases identify those, but in general their spaces have different sizes. Existence of symmetries is a constraint on the system.

The models with the maximal amount of symmetries, conservations laws etc are of special significance. Usually sub-maximal symmetric models play an important role in applications too.

The setup for the enumeration problem can be: micro-local (the objects are defined locally in the space of jets), local (with respect to the manifold) or global. While the first two cases are often the same (provided we impose the requirement of algebraic behavior for the solutions, e.g. considering polynomial in momenta first integrals), the latter two are always different: the global existence can have strong topological implications.

Key words and phrases. Overdetermined systems, compatibility, symmetric models, polynomial integrals, Tanaka algebra. 
For instance, the number of Killing vector fields locally on 2-disk $D^{2}$ can be only 0,1 or 3 , but globally on $T^{2}$ it is 0,1 or 2 . Similarly, the number of quadratic integrals locally is $1,2,3,4$ or 6 [D], but globally it can be 2 or 6 on $S^{2}$ and 2 or 3 on $T^{2}$ [Kol, Ki]. In any case the maximal number means constant curvature.

We will restrict exclusively to local problems, so all differential equations and their solutions are defined locally near a regular point.

The following phenomenon is known classically: the maximal and the next after it (sub-maximal) amounts of the objects we count (symmetries, integrals etc) usually have a gap between them.

For instance the maximal amount of point transformations of 2 nd order scalar ODEs is 8 (trivializable ODEs with $\mathrm{sl}_{3}$ algebra of symmetries), elsewise there are no more than 3 symmetries according to A. Tresse classification $\left[\mathrm{Tr}, \mathrm{K}_{2}\right.$.

In some cases this gap (we stick to usage of this term, resembling "spectral gap"; in Russian tradition the word "lacune" is adopted) can be explained by the structure theory of simple Lie groups, but in some others the algebraic explanation is lacking.

In this paper we will demonstrate the gap phenomenon in a number of examples. In the particular case, when the geometric structures are encoded by vector distributions, we remark that the maximal symmetric model is unique and the maximal symmetry algebra is graded. Then we give a tool to control the gap. Namely, using recent advances in the Tanaka theory, we clarify the behavior of symmetric models and we relate this to algebraic and homological methods.

In general the reason for appearance of the gap and its size remains an interesting open problem.

The paper is organized as follows. In Section 1 we recall the general setup from geometric theory of differential equations, giving the method to measure dimensional characteristics we discuss. In Section 2 we discuss the automorphisms of geometric structures. Most results here are classical. In Section 3 we consider the problem of integrability of geodesic flows, and present old and new knowledge on the number of second and higher order integrals.

Then in Section 4 we recall basics from the Tanaka theory together with a recent progress, and formulate a method to find sub-maximal symmetric structures. This will be applied in Section 5 to the geometry of rank 2 distributions in manifolds of dimension 5 and 6 . The former is known since E. Cartan [C] and we give a new interpretation of his equations, while the latter will contain new results and we sketch their higher-dimensional analogs.

We finish with conclusion, summarizing the results of the paper. 
ACKNOWLEDGMENT. This research was partially supported by the grant DAAD project Nr. 208068. I would like to thank Vladimir Matveev and other organizers of the conference "Finite Dimensional Integrable Systems in Geometry and Mathematical Physics 2011" in Jena for hospitality. Some calculations in the last section were performed using MAPLE package DifferentialGeometry.

\section{Solution Space of Differential EQuations}

The systems discussed in the Introduction can be re-cast into counting dimensions of the solutions spaces for certain (systems of) differential equations. For our purposes we can restrict to linear PDEs.

A differential equation $\mathcal{E}$ is represented geometrically as a submanifold in the space of jets $J^{k}(M, N)$, where $M$ is the space of independent variables $x=\left(x^{1}, \ldots, x^{n}\right)$ and $N$ is the space of dependent variables $u=\left(u^{1}, \ldots, u^{m}\right)$. A choice of these coordinates on $J^{0}=M \times N$ yields canonical coordinates on the space $J^{k}:\left(u_{\sigma}^{j}\right)$, where multi-index $\sigma=\left(i_{1}, \ldots, i_{n}\right)$ has length $|\sigma|=\sum_{a=1}^{n} i_{a} \leq k$.

A system $\mathcal{E}$ of differential equations on $u=u(x)$ can be considered as a submanifold in jets. We will assume regularity, i.e. the projection maps $\pi_{k, i}: J^{k} \rightarrow J^{i}$ have constant ranks when restricted to $\mathcal{E}$ and its prolongations. If $\mathcal{E}$ is given by differential equations $F_{j}=0$ of orders $k_{j}$, then the prolongation to jet-order $l$ is given as the locus $\mathcal{E}_{l} \subset J^{l}(M, N)$ of equations $\mathcal{D}_{\sigma} F_{j}=0$, where $\mathcal{D}_{\sigma}$ denotes the total derivative by multiindices $\sigma$, and the maximal amount of differentiations is $|\sigma| \leq l-k_{j}$.

The symbols of $\mathcal{E}$ are defined as $g_{i}=\operatorname{Ker}\left(d \pi_{i, i-1}: T \mathcal{E}_{i} \rightarrow T \mathcal{E}_{i-1}\right)$. If $x_{i} \in \mathcal{E}_{i}$ and $\pi_{i}\left(x_{i}\right)=x \in M$, we can identify $g_{i}\left(x_{i}\right) \subset S^{i} T_{x}^{*} M \otimes T_{x} N$.

In general $\operatorname{dim} g_{i}$ as a function of $i$ grows in accordance with the Hilbert polynomial $[\mathrm{KL}$, that can be calculated by the characteristic variety of $\mathcal{E}$. System $\mathcal{E}$ is said to be of finite type if $g_{i}(x)=0$ for large $i$ and all $x$ (this holds iff the complex characteristic variety is empty).

In other words, $\mathcal{E}$ is of finite type if for some jet-level $\pi_{l, l-1}: \mathcal{E}_{l} \rightarrow \mathcal{E}_{l-1}$ is a diffeomorphism, i.e. all the jets of $u$ of order $l$ are expressed through the lower order jets. This uses regularity assumptions; if we relax the latter then the conclusion holds on an open dense set (but $g_{i}(x)=0$ for large $i$ should hold uniformly in $x \in M)$. In this case the solution space can be parametrized by the Cauchy data.

Theorem 1. Dimension of the solution space of a finite type system $\mathcal{E}$ is bounded as follows (the sum is finite)

$$
\operatorname{dim} \operatorname{Sol}(\mathcal{E}) \leq \sup _{M} \sum_{i} \operatorname{dim} g_{i}(x) .
$$

For the regular case, when $\operatorname{dim} g_{i}(x)$ are constants, this is a folklore classical result; for the general case we refer to $\mathrm{K}_{3}$ (it is possible to 
use inf instead of sup in the right hand side; the result also holds for analytic $\mathcal{E}$ which have finite type almost everywhere).

Equality in (1) is attained only in the case $\mathcal{E}$ is formally integrable, i.e. $\mathcal{E}$ is regular (this assumption we adopt from now on) and $\pi_{i, i-1}$ : $\mathcal{E}_{i} \rightarrow \mathcal{E}_{i-1}$ is a vector bundle. The latter means that all compatibility conditions of $\mathcal{E}$ hold true.

Notice that if $\mathcal{E}$ is compatible, the symbols of the prolongations $g_{l}$ can be calculated from the symbols of the equation $g_{k}$ by purely algebraic prolongations $g_{l}=g_{k}^{(l-k)}=g_{k} \otimes S^{l-k} T_{x}^{*} M \cap S^{l} T_{x}^{*} M \otimes T_{x} N$, but in general (non-integrable) case the algebraic prolongations can exceed the actual symbol spaces.

Consider a family of equations $\mathcal{E}_{\alpha}$ depending on a numeric or functional parameter $\alpha$. The parametrization will be assumed algebraicdifferential. For instance $\mathcal{E}$ can be the Lie equation describing symmetries of a geometric object and $\alpha$ can parametrize these objects (like Riemannian metrics or distributions of prescribed dimensions/type). We will assume that the symbol spaces $g_{i}$ calculated algebraically have the same dimensions independent of the parameter $\alpha$.

In this paper we we would like to understand what are the maximal dimensions $\operatorname{dim} \operatorname{Sol}\left(\mathcal{E}_{\alpha}\right)$ and what are the respective structures. This corresponds to the parameters $\alpha$ for which $\mathcal{E}_{\alpha}$ is formally (and so by finite type condition also locally) integrable.

It turns out that the possible dimensions of $\operatorname{dim} \operatorname{Sol}\left(\mathcal{E}_{\alpha}\right)$ usually have a gap between the maximal value and the next one. The basic reason behind this is the following. Non-maximality means that some compatibility conditions fail, and so new equations shall be added to the collection $\left\{F_{j}=0\right\}$. This reduces dimension of some symbol space $g_{i}$ and its prolongations, and so the right hand side of (11) decreases.

We are going to demonstrate this effect with examples.

\section{Automorphisms of Classical Geometric STRUCtures}

Riemannian structures or pseudo-Riemannian structures on a manifold $M$ always have finite-dimensional isometry groups, which are solutions to the Lie equation $\mathcal{E}=\left\{[\varphi]_{x}^{1}: d_{x} \varphi(g)=g\right\} \subset J^{1}(M, M)$. The corresponding Lie algebra consists of vector fields $\xi$ satisfying $L_{\xi}(g)=0$. The symbols of this equation are (we identify $T M \simeq T^{*} M$ via $g$ )

$$
g_{0}=T_{x} M, g_{1}=\Lambda^{2} T_{x}^{*} M, g_{2}=g_{1}^{(1)}=0,
$$

whence $\operatorname{dim} g_{0}=n, \operatorname{dim} g_{1}=\frac{n(n-1)}{2}$. Thus the system has finite type on the level of 2-jets, $\pi_{2,1}: \mathcal{E}_{2} \stackrel{\sim}{\rightarrow} \mathcal{E}_{1}$, and the maximal dimension of the 
solution space is

$$
\mathfrak{S}_{\max }=\sum \operatorname{dim} g_{i}=\frac{n(n+1)}{2}
$$

This is realized precisely when $\mathcal{E}$ is compatible, which corresponds to the spacial forms (constant sectional curvature), so that locally $M$ is round sphere $S^{n}$, Euclidean space $\mathbb{R}^{n}$ or the Lobachevsky space $L^{n}$. The symmetry algebras are resp. $\mathfrak{s o}(n+1), \mathfrak{s o}(n) \ltimes \mathbb{R}^{n}$ or $\mathfrak{s o}(1, n)$.

Let us discuss the submaximal dimension in the Riemannian case. The stabilizer of a point $x_{0}$ is a subalgebra in $\mathfrak{s o}(n)$. If it is full, the translational part at $x_{0}$ should be either of dimension $n$ or zero. In the first case we get the constant curvature, in the second - surface of revolution. If the isotropy group is proper, then (as maximal) it is conjugated to $\mathfrak{s o}(n-1)$, provided $n \neq 4$. Thus the metric again gives the surface of revolution $d s^{2}=d r^{2}+f(r)^{2} d s_{n-1}^{2}$. Translation part is full only if the sectional curvature for $d s_{n-1}^{2}$ is constant and to exclude the maximal case, we suppose the curvature is non-zero. The gap is thus $(n-1)$ for $n \neq 2$, 4 , i.e. the sub-maximal symmetry algebra of a Riemannian metric $g$ has dimension

$$
\mathfrak{S}_{\text {sub.max }}=\frac{n(n-1)}{2}+1
$$

The sub-maximal homogeneous Riemannian spaces were described by K.Yano, M.Obata and N.Kuiper for $n \geq 4$, see ref. in [Ko, YK], and by E.Cartan in dimension 3 and I.Egorov in dimension $4\left[\mathrm{E}_{1}\right]$.

In dimension 2 the submaximal case has dimension 1 (this result belongs to Darboux and Könings [D]), and in dimension 4 the submaximal case has dimension 8 - in both cases the gap is $2\left[\mathrm{E}_{2}\right]$.

In pseudo-Riemannian case the situation is more complicated. The maximal case still corresponds to the manifold of constant sectional curvature $M^{n}(c)$, i.e. locally Minkovsky or Wolf space. The submaximal case has the same dimension of the symmetry algebra in the case of Lorenzian signature and $n \neq 3,5$, but the amount of submaximal cases is greater. According to $[\mathrm{P}]$ for $n>5, n \neq 7$ locally they are: products $M^{n-1}(c) \times \mathbb{R}, \epsilon$-spaces and Egorov spaces.

Affine structure is given by a choice of affine connection on $M$. The Lie equation for symmetries $\mathcal{E} \subset J^{2}(M, M)$ at some point $x_{2} \in \mathcal{E}$ with $\pi_{2}\left(x_{2}\right)=x$ has symbols $g_{0}=T_{x} M, g_{1}=T_{x}^{*} M \otimes T_{x} M, g_{2}=0$.

Thus the symmetry algebra of the affine connection has dimension at most

$$
\mathfrak{S}_{\max }=n+n^{2}
$$


The equality is attained iff $\mathcal{E}$ is compatible, and this happens precisely when $M$ is the usual affine space with flat connection [Ei]. The submaximal case for $n \geq 4$ has dimension

$$
\mathfrak{S}_{\text {sub.max }}=n^{2} \text {. }
$$

Almost complex structure is a field of operators $J$ on $T M$ satisfying $J^{2}=\mathbf{- 1}$. If the Nijenhuis tensor $N_{J}$ vanishes, the structure is integrable and the local symmetry pseudogroup is infinite-dimensional. To guarantee finite-dimensionality one imposes a non-degeneracy condition on the Nijenhuis tensor $\left[\mathrm{K}_{4}\right.$. If $n=\operatorname{dim}_{\mathbb{C}} M>2$, this says that the image $N_{J}\left(\Lambda^{2} T M\right)$ is the whole $T M$. For $n=2$ it is enough to claim that the image be a non-holonomic rank 2 distribution.

Provided the dimension of the symmetry group is finite what is its maximal value? We know the definite answer for $n=3$. According to $\mathrm{K}_{4}$ the maximal symmetric non-degenerate almost complex structure is the Calabi structure $J$ on $S^{6}$, the symmetry group being $G_{2}$. We do not know the submaximal model for certain, but expect its symmetry algebra to be 9-dimensional (then the gap will be equal to 5).

Conformal structure is given by a choice of conformal class of (pseudo-)Riemannian metric. The Lie equation $\mathcal{E} \subset J^{1}(M, M)$ for $n>2$ has symbols $g_{0}=T_{x} M, g_{1}=\operatorname{co}(n)=\mathbb{R} \oplus \mathfrak{s o}(n) \simeq \mathbb{R} \oplus \Lambda^{2} T_{x}^{*} M$, $g_{2}=T_{x}^{*} M$ and $g_{3}=0$. Thus

$$
\mathfrak{S}_{\max }=\frac{(n+1)(n+2)}{2} \text {. }
$$

Equality is attained in conformally flat case (loc. Euclidean space $\mathbb{R}^{n}$ ). The same is true for the signature $(p, n-p) \sqrt{1}$.

Projective structure can be given as a projective class of torsion free connection on $M$. The symbols of its Lie equation are $g_{0}=T_{x} M$, $g_{1}=T_{x}^{*} M \otimes T_{x} M, g_{2}=T_{x}^{*} M$ and $g_{3}=0$. Thus

$$
\mathfrak{S}_{\max }=n^{2}+2 n \text {. }
$$

Equality is attained in projectively flat case $\left(\right.$ loc. $\left.\mathbb{R P}^{n}\right)$, see $[\mathrm{Ko}$. We do not know submaximal cases for conformal and projective structures.

Closely related to projective equivalences are geodesic equivalences between Riemannian manifolds. The counting function for the latter - the degree of geodesic mobility - has lacunarity similar to that in the theory of motion. See $\left[\mathrm{KM}^{2} \mathrm{~S}\right]$ and the references therein for complete description of gaps in the distribution of the degree of geodesic

\footnotetext{
${ }^{1}$ Added in proof: after discussion with M.Dunajski and K.Melnik it became clear that the sub-maximal model in dimension $n=4$ and neutral signature is given by the so-called pp-waves metric. This is the anti-self-dual twist free, constant curvature solution of the Einstein equations $\mathrm{Du}$. Its symmetry algebra has dimension 9 , and so the gap is 6 in this case.
} 
mobility. The maximal and sub-maximal degrees of geodesic mobility for $n>2$ are

$$
\mathfrak{S}_{\max }=\frac{(n+1)(n+2)}{2}, \quad \mathfrak{S}_{\text {sub.max }}=\frac{(n-1)(n-2)}{2}+1 .
$$

Thus the gap is $3 n-1$. The dimension of concircular fields, which are projective analogs of the Killing fileds, have gap 3 as for them

$$
\mathfrak{S}_{\max }=n+1, \quad \mathfrak{S}_{\text {sub.max }}=n-2 .
$$

That degree of geodesic mobility exceeds 2 is closely related to integrability of geodesic flows [MT, which is discussed in the next section (in particular for $n=2$, when the degree of geodesic mobility equals $\operatorname{dim} Q_{2}$, see (2), we observe that the maximal case has the same dimension as prescribed above, namely $\mathfrak{S}_{\max }=6$, while the sub-maximal case has dimension $\mathfrak{S}_{\text {sub.max }}=4$ ).

\section{INTEGRALS OF GEODESIC FLOWS}

Let $(M, g)$ be a Riemannian or pseudo-Riemannian manifold. The geodesic flow of $g$ is the dynamical system on $T^{*} M$ (with the canonical symplectic form) given by the Hamiltonian $H=\frac{1}{2}\|p\|_{g}^{2}=\frac{1}{2} g^{i j}(x) p_{i} p_{j}$. Its integrability via functions analytic in momenta is equivalent, by Whittaker's theorem, to existence of polynomial in momenta integrals. Since $H$ is quadratic we can restrict to integrals of pure degree $d$.

Consider the vector space $(\{$,$\} denotes the standard Poisson bracket)$

$$
Q_{d}(g)=\left\{F \in C^{\infty}\left(T^{*} M\right):\{H, F\}=0 \text { and } \operatorname{deg} F=d\right\} .
$$

We will be interested in its dimension depending on $g$. The whole algebra of polynomial integrals $Q(g)=\oplus Q_{d}(g)$ is graded and Poisson, satisfying $\left\{Q_{k}, Q_{l}\right\} \subset Q_{k+l-1}$.

Linear integrals, also known as Killing vector fields, have the form $F=u^{k}(x) p_{k}$. The coefficients of the quadratic expression $\{H, F\}=0$

give a system $\mathcal{E}$ of $\frac{n(n+1)}{2}$ equations on $n$ unknown functions. The symbols of $\mathcal{E}$ have the following dimensions (interpreted as the number of free jets, or as the amount of Cauchy data):

$$
\operatorname{dim} g_{0}=n, \quad \operatorname{dim} g_{1}=n^{2}-\frac{n(n+1)}{2}=\frac{n(n-1)}{2}, \quad \operatorname{dim} g_{2}=0 .
$$

Its easy to see that this is the Lie equation for the Riemannian metric $g$ - the case discussed in $\$ 2$.

Solution to this $\mathcal{E}$ gives the Lie algebra $\operatorname{sym}(g)=Q_{1}(g)$. The other spaces $Q_{d}(g)$ are not Lie algebras, but are modules over $Q_{1}(g)$.

Quadratic integrals, also known as Killing-Stäckel 2-tensors, have the form $F=u^{k l}(x) p_{k} p_{l}$. The coefficients of the cubic expression 
$\{H, F\}=0$ give a system $\mathcal{E}$ of $\frac{n(n+1)(n+2)}{6}$ equations on $\operatorname{dim} g_{0}=\frac{n(n+1)}{2}$ unknowns. The higher symbols of $\mathcal{E}$ have the following dimensions:

$$
\operatorname{dim} g_{1}=\frac{n\left(n^{2}-1\right)}{3}, \quad \operatorname{dim} g_{2}=\frac{n^{2}\left(n^{2}-1\right)}{12}, \quad \operatorname{dim} g_{3}=0 .
$$

Thus the system has finite type on the level of 3 -jets, $\pi_{3,2}: \mathcal{E}_{3} \stackrel{\sim}{\rightarrow} \mathcal{E}_{2}$, and the maximal dimension of the solution space is

$$
\mathfrak{S}_{\max }=\sum \operatorname{dim} g_{i}=\frac{(n+1)^{2}\left((n+1)^{2}-1\right)}{12} .
$$

This is realized precisely when $\mathcal{E}$ is compatible, which corresponds to the spacial forms (it works also for the pseudo-Riemannian case).

In fact, the maximal space of quadratic integrals $Q_{2}=S^{2} Q_{1}$. This means that quadratic integrals are linear combinations of the products of the linear ones. For instance, for the flat case $Q_{1}=\left\langle p_{k}, r_{i j}=x_{i} p_{j}-\right.$ $\left.x_{j} p_{i}\right\rangle$, so $Q_{2}$ is generated by $g_{0}=\left\langle p_{i} p_{j}\right\rangle, g_{1}=\left\langle L_{i j k}=r_{i j} p_{k}\right\rangle$ (relations are $L_{i j k}=-L_{j i k}$ and $\left.L_{i j k}+L_{j k i}+L_{k i j}=0\right)$ and $g_{2}=\left\langle R_{i j k l}=r_{i j} r_{k l}\right\rangle$ (relations are the same as for algebraic curvature tensors).

Proposition 2. Submaximal symmetric Riemannian metrics have dimension of the solution space $Q_{2}$ given by

$$
\mathfrak{S}_{\text {sub.max }} \geq \frac{n(n+1)}{2}+\frac{n^{2}\left(n^{2}-1\right)}{12} .
$$

In fact we expect that the above is the submaximal number of quadratic integrals. The claim follows from the following

Lemma 1. For the metric $g=x_{1} \sum_{1}^{n} d x_{i}^{2}$

$$
\operatorname{dim} Q_{2}(g)=\frac{n(n+1)}{2}+\frac{n^{2}\left(n^{2}-1\right)}{12} .
$$

Proof. The Hamiltonian is $H=\frac{1}{x_{1}} \sum p_{i}^{2}$. Let $F=\sum b_{i j} p_{i} p_{j}$ be a quadratic integral. The condition $\{H, F\}=0$ is equivalent to the PDE system on functions $b_{i j}=b_{i j}(x)$. The coefficient of $p_{1}^{3}$ gives

$$
\frac{\partial b_{11}}{\partial x_{1}}=-\frac{b_{11}}{x_{1}} \Rightarrow b_{11}=\frac{\beta\left(x_{2}, \ldots, x_{n}\right)}{x_{1}} .
$$

The coefficients of $p_{1}^{2} p_{i}$ for $i>1$ give

$$
2 x_{1} \frac{\partial b_{1 i}}{\partial x_{1}}+b_{1 i}+\frac{\partial \beta}{\partial x_{i}}=0 \Rightarrow b_{1 i}=\frac{\sigma_{i}\left(x_{2}, \ldots, x_{n}\right)}{\sqrt{x_{1}}}-\frac{\partial \beta}{\partial x_{i}} .
$$

The coefficients of $p_{1} p_{i}^{2}$ and $p_{1} p_{i} p_{j}$ for $i, j>1$ give

$$
b_{i j}=\tau_{i j}\left(x_{2}, \ldots, x_{n}\right)-2 \sqrt{x_{1}}\left(\frac{\partial \sigma_{i}}{\partial x_{j}}+\frac{\partial \sigma_{j}}{\partial x_{i}}\right)+2 x_{1} \frac{\partial^{2} \beta}{\partial x_{i}^{2}}+\frac{\beta}{x_{1}} \delta_{i j} .
$$


Substituting these expressions into the coefficients of $p_{i}^{3}, p_{i}^{2} p_{j}$ and $p_{i} p_{j} p_{k}$ and separating different powers of $x_{1}$ we obtain that $\beta$ is arbitrary inhomogeneous quadric, $\sigma=0$ and $\tau$ correspond to the (maximal) space of Killing 2-tensors in $(n-1)$ dimensions. Since the inhomogeneous quadrics in $(n-1)$ dimensions are bijective with homogeneous quadrics in $n$ dimensions, we are done.

Notice that for the above structure $\operatorname{dim} Q_{1}(g)=\frac{n(n-1)}{2}, n>1$, so that for $n>2$ it is not sub-maximal for the dimension of Killing fields. Let us consider sub-maximal metrics for $\operatorname{dim} Q_{1}$; they turn out to be not submaximal for the dimension of Killing 2-tensors.

Lemma 2. For $n>2$ and the metric $g=d x_{1}^{2}+d s_{n-1}^{2}$, where the latter is a metric on $D^{n-1}\left(x_{2}, \ldots, x_{n}\right)$ of constant sectional curvature $c \neq 0$,

$$
\operatorname{dim} Q_{2}(g)=1+\frac{n(n-1)}{2}+\frac{n^{2}\left(n^{2}-1\right)}{12} .
$$

Proof. We will consider only the case of positive curvature $c>0$. Using stereographic coordinates on $M^{n-1}(c)$ the Hamiltonian on $T^{*} M$ writes

$$
H=p_{1}^{2}+\left(R^{2}+x_{2}^{2}+\cdots+x_{n}^{2}\right)^{2}\left(p_{2}^{2}+\cdots+p_{n}^{2}\right) .
$$

The Killing fields (written as functions on $T^{*} M$ ) are

$$
K_{1}=p_{1}, \quad K_{i}^{0}=\left(R^{2}-\sum_{2}^{n} x_{j}^{2}\right) p_{i}+2 x_{i} \sum_{2}^{n} x_{j} p_{j}, \quad K_{i j}^{1}=x_{i} p_{j}-x_{j} p_{i}
$$

(the indices $i, j$ run from 2 to $n$ ).

Write the quadratic integral $F=a p_{1}^{2}+p_{1} \sum_{2}^{n} b_{i} p_{i}+\sum_{i, j>1} c_{i j} p_{i} p_{j}$. The coefficients of $p_{1}^{3}$ in the equation $\{H, F\}=0$ yields $\frac{\partial a}{\partial x_{1}}=0$. Then the coefficients of $p_{1}^{2} p_{i}$ yields $b_{i}=b_{i}^{0}-\left(R^{2}+x_{2}^{2}+\cdots+x_{n}^{2}\right) x_{1} \frac{\partial a}{\partial x_{i}}$, where $b_{i}^{0}=b_{i}^{0}\left(x_{2}, \ldots, x_{n}\right), i>1$.

The coefficients of $p_{i} p_{j} p_{k}$ with $i, j, k>1$ yield the condition that $\sum_{i, j>1} c_{i j} p_{i} p_{j}$ is a Killing 2-tensor of the metric $d s_{n-1}^{2}$. Since the latter has constant sectional curvature, we conclude that this contribution to $F$ is generated by pairwise products of Killing fields in $(n-1)$ dimension:

$$
\left\langle\sum_{i, j>1} c_{i j} p_{i} p_{j}\right\rangle=S^{2}\left\langle K_{i}^{0}, K_{i j}^{1}\right\rangle
$$

Substituting this and evaluating the coefficient of $p_{1} p_{i} p_{j}$ we obtain certain expressions linear in $x_{1}$, for which free terms mean that $\sum_{2}^{n} b_{i}^{0} p_{i}$ is a Killing fields for $d s_{n-1}^{2}$. Thus it is a linear combination of $K_{i}^{0}, K_{i j}^{1}$.

The coefficients of $x_{1}$ give a system on $a=a\left(x_{2}, \ldots, x_{n}\right)$ of the second order in Frobenius form (its second symbol vanish $g_{2}=0$ ). But it is inconsistent - the compatibility conditions give $a=$ const. The claim follows. 
Let us notice that in the case of Lemma 2 we have $Q_{2}(g)=S^{2} Q_{1}(g)$, but this equality is wrong in the case of Lemma 1 .

Cubic and higher degree integrals. In any order $d$ and dimension $n$ the quantity $\operatorname{dim} Q_{d}(g)$ attains maximum precisely when $g$ is a spacial form. In addition $Q_{d}(g)=S^{d} Q_{1}(g)$ for such metrics $g$.

Almost nothing is known about submaximal cases, except for $n=2$. Consider the latter case. It was shown in $\mathrm{K}_{1}$ that for $d=3$ and $g$ being of non-constant curvature $\operatorname{dim} Q_{3}(g)<7$ (for the maximal case this dimension is 10). Thus the gap is at least 4 , and we conjectured it is 6 (i.e. submaximal $\operatorname{dim} Q_{3}=4$ the same as submaximal $\operatorname{dim} Q_{2}$ ).

This conjecture was proved under additional assumption that $g$ has a Killing field in MS. The assumption looks natural - one expects from experience with degree $d=2$ that the metric of submaximal type possesses an additional linear integral.

Let us notice that the success of the above approach is related to the fact that given $H$, the system $\{H, F\}=0$ on coefficients of $F$ is overdetermined and of finite type. If we consider both $H$ and $F$ as unknowns, the situation changes (we treat only the local case).

Denote by $\overline{\mathcal{E}}$ the system on coefficients of $H, F$. If one of $k=\operatorname{deg} H$ or $l=\operatorname{deg} F$ equals to 1 , then $\overline{\mathcal{E}}$ is underdetermined (implying existence of functional families of solutions). So we assume $l \geq k>1$ and use the identity $\operatorname{deg}\{H, F\}=k+l-1$.

In dimension $n=2$, due to existence of isothermal coordinates, the system is determined for all $k, l$. In particular, for $k=2$ it has the structure of semi-Hamiltonian system of hydrodynamic type [BM].

For $n=3$ the system $\overline{\mathcal{E}}$ is underdetermined for $k=2, l=2,3$ and determined for $(k, l)=(2,4)$. For $n=4$ it is determined for $(k, l)=$ $(2,2)$. For all other parameters and for $n \geq 5 \overline{\mathcal{E}}$ is overdetermined (so dimensional restrictions on its solution space are expected).

\section{TANAKA THEORY: OLD AND NEW}

Tanaka theory [T] describes symmetries (automorphisms) of vector distributions $\Delta \subset T M$. With every such distribution one associates the graded Tanaka algebra $\mathfrak{g}$ defined as follows.

Define the weak derived flag $\left\{\Delta_{i}\right\}$ through the module of its sections by $\Gamma\left(\Delta_{i+1}\right)=\left[\Gamma(\Delta), \Gamma\left(\Delta_{i}\right)\right]$ with $\Delta_{1}=\Delta$. We assume that our distribution is completely non-holonomic, i.e. $\Delta_{k}=T M$ for some $k$, and that $\Delta$ is regular, i.e. ranks of $\Delta_{i}$ are constant along $M$.

The quotient sheaf $\mathfrak{m}=\oplus_{i<0} g_{i}, g_{i}=\Delta_{-i} / \Delta_{-i-1}$ has a natural structure of a graded nilpotent Lie algebra at any point $x \in M$. The bracket on $\mathfrak{m}$ is induced by the commutator of vector fields on $M$. Distribution 
$\Delta$ is called strongly regular if $\mathfrak{m}(x)$, as a Lie algebra, does not depend on the choice of $x \in M$.

The Tanaka prolongation $\mathfrak{g}=\mathfrak{m} \oplus \mathfrak{g}_{+}=\mathfrak{g}_{-k} \oplus \ldots \mathfrak{g}_{-1} \oplus \mathfrak{g}_{0} \oplus \mathfrak{g}_{1} \oplus \ldots$ is the maximal graded Lie algebra with negative graded part equal to $\mathfrak{m}$. The non-negative part $\mathfrak{g}_{+}$is a subalgebra, and it a-priori can be infinite-dimensional. The graded components can be calculated algorithmically, see $[\mathrm{T}, \mathrm{AK}]$. For instance $\mathfrak{g}_{0}$ is the space of grading preserving derivations of $\mathfrak{m}$.

For strongly regular distributions N. Tanaka constructed an absolute parallelism on the prolongation manifold, and this gives the way to define curvature and flatness [T]. In particular, it shows the standard model $\left(\operatorname{Exp}(\mathfrak{m}), \mathfrak{g}_{-1}\right)$ gives the maximal symmetric distribution (among strongly regular distributions) with the symmetry algebra $\mathfrak{g}$.

The condition of strong regularity was removed in $\left.\mathrm{K}_{3}\right]$. The following statement is a combination of results in $\mathrm{K}_{3}, \mathrm{AK}$ (dimensional part is a paraphrase of Theorem 1, in the first part of the theorem sup can be changed to inf).

Theorem 3. Let $\Delta$ be of finite type, i.e. for some $k$ we have: $\mathfrak{g}_{i}(x)=0$, for all $i \geq k, x \in M$. Then its symmetry algebra is majorized by the Tanaka algebra $\mathfrak{g}$ in the sense

$$
\operatorname{dim} \operatorname{sym}(\Delta) \leq \sup _{M} \sum_{i} \operatorname{dim} \mathfrak{g}_{i}(x) .
$$

This inequality is an equality if and only if the distribution $\Delta$ is flat.

Thus the maximal symmetry model is unique (isomorphic to the standard model), which is not the case with maximal cases for other geometric structures.

The following statement re-phrases Theorem 3 from $\mathrm{K}_{3}$.

Theorem 4. Let $h$ be a Lie algebra symmetry of a distribution $\Delta$ on a manifold $M$, and let $\mathfrak{g}$ be the Tanaka algebra of $\Delta$. Then there exists a Lie algebra compatible filtration $F_{i}$ of $h$ such that the corresponding graded Lie algebra is a subalgebra of the Tanaka algebra: $\mathfrak{h} \subset \mathfrak{g}$.

This is to be compared to the following result of R. Zimmer [Z] (which uses the assumption $g_{1}(x) \subset \mathfrak{s l}\left(T_{x} M\right)$ instead of grading).

Theorem 5. Let $M$ be a compact group, $G$ a real algebraic group acting on $M$ by volume-preserving transformations and $P$ be a $G$-structure. Then Lie algebra $\mathfrak{h}=\operatorname{sym}(P)$ embeds into $\mathfrak{g}=\operatorname{Lie}(G)$.

Theorem 4 can be applied as follows.

Corollary 6. Any sub-maximal symmetric case is obtained via the following construction. 
Consider a subalgebra $\mathfrak{h} \subset \mathfrak{g}$ and let $h$ be a filtered Lie algebra that cannot be monomorphically mapped into $\mathfrak{g}$ (as a filtered algebra), but whose associated graded Lie algebra equals $\mathfrak{h}$.

Suppose that $h$ has a subalgebra $h_{0}$ of dimension $\operatorname{dim} \mathfrak{g}-\operatorname{dim} M$ and that for a vector subspace $\Pi \subset h$ of dimension equal to $\operatorname{rank}(\Delta)$ we have: $\left[h_{0}, \Pi\right] \subset h_{0}+\Pi, h_{0} \cap \Pi=0$.

If the iterated brackets of $h_{0}+\Pi$ generate $h$, then the homogeneous space $\mathrm{H} / \mathrm{H}_{0}$ (where $\mathrm{H}, \mathrm{H}_{0}$ are the corresponding Lie groups) possesses $H$-invariant nonholonomic distribution corresponding to $\Pi$.

We call such an algebra $h$ a deformation of $\mathfrak{h}$. Notice that if $h \subset \mathfrak{g}$ then the symmetry algebra of the distribution on $H / H_{0}$ will be $\mathfrak{g}$, i.e. the maximal instead of sub-maximal case.

First obstructions to deformations of Lie algebra structure are given by $H^{2}(\mathfrak{h}, \mathfrak{h})$. These cohomology groups can be however non-zero even in rigid cases (this is what often happens when $\mathfrak{h}$ is not semi-simple). We will see an example of this in the next section.

\section{SyMmetriES OF RANK 2 DistRIBUTIONS}

Consider rank 2 distributions, at first in 5-dimensional space. According to Goursat $G$ ] they are encoded as Monge underdetermined ODEs

$$
y^{\prime}=F\left(x, y, z, z^{\prime}, z^{\prime \prime}\right) .
$$

The equation-manifold $\mathbb{R}^{5}\left(x, y, z, z_{1}, z_{2}\right)$ is equipped with the Pfaffian system $\left\{d z-z_{1} d x, d z_{1}-z_{2} d x, d y-F d x\right\}$ and the rank 2 distribution $\left\langle\mathcal{D}_{x}=\partial_{x}+z_{1} \partial_{z}+z_{2} \partial_{z_{1}}+F \partial_{y}, \partial_{z_{2}}\right\rangle$ is dual to it.

Internal symmetries of (3) are by definition the symmetries (automorphisms) of this distribution.

Condition $F_{z_{2} z_{2}} \neq 0$ guarantees that the symmetry algebra is finitedimensional. In this case E. Cartan C showed that the dimension is bounded by 14 , and in the case of equality the maximal group is $G_{2}$. The maximal symmetric model is unique up to equivalence and it is given by the celebrated Hilbert-Cartan equation

$$
y^{\prime}=\left(z^{\prime \prime}\right)^{2} .
$$

What about sub-maximal cases? They all are given by the following Monge equations (in [C] overdetermined involutive 2nd order PDE systems on the plane were classified, but it is not difficult to establish the equivalence):

$$
y^{\prime}=\left(z^{\prime \prime}\right)^{m} .
$$

Here $m=0,1$ corresponds to the case of Engel distribution in $\mathbb{R}^{4}$ which has the contact pseudogroup of symmetries; if $2 m-1 \in\left\{ \pm \frac{1}{3}, \pm 3\right\}$ the equation is equivalent to (44) and its symmetry is the exceptional Lie 
group $G_{2}$; for other cases the group of symmetries $\mathfrak{g}$ has dimension 7 ( $m \in \mathbb{R}$ is the only invariant of both the group and the equation) and is given in generators as follows:

$$
\begin{gathered}
\operatorname{sym}\{(15)\}=\left\langle W_{1}=\partial_{x}, W_{2}=\partial_{y}, W_{3}=\partial_{z}, W_{4}=x \partial_{x}+y \partial_{y}+2 z \partial_{z}+z_{1} \partial_{z_{1}},\right. \\
W_{5}=x \partial_{z}+\partial_{z_{1}}, W_{6}=m y \partial_{y}+z \partial_{z}+z_{1} \partial_{z_{1}}+z_{2} \partial_{z_{2}}, W_{7}= \\
\left.z_{2}^{m-1} \partial_{x}+(m-1) \int z_{2}^{2 m-2} d z_{2} \cdot \partial_{y}+\left(z_{1} z_{2}^{m-1}-\frac{1}{m} y\right) \partial_{z}+\left(1-\frac{1}{m}\right) z_{2}^{m} \partial_{z_{1}}\right\rangle .
\end{gathered}
$$

Abstractly the Lie algebra structure reads off from the structure equations on p.169 $\mathrm{C}$. In the basis $\left\langle X_{1}, X_{2}, X_{3}, X_{4}, X_{5}, Y_{1}, Y_{2}\right\rangle$ dual to the Cartan coframe $\left\langle\omega_{1}, \omega_{2}, \omega_{3}, \omega_{4}, \omega_{5}, \varpi_{1}, \varpi_{2}\right\rangle$ the non-trivial commutators are

$$
\begin{gathered}
{\left[X_{1}, Y_{1}\right]=2 X_{1},\left[X_{2}, Y_{2}\right]=X_{1},\left[X_{3}, X_{4}\right]=X_{1},} \\
{\left[X_{2}, Y_{1}\right]=X_{2},\left[X_{3}, Y_{1}\right]=X_{3},\left[X_{4}, Y_{1}\right]=X_{4},\left[Y_{2}, Y_{1}\right]=Y_{2},} \\
{\left[X_{2}, X_{5}\right]=\left(I X_{3}+Y_{2}\right),\left[X_{3}, X_{5}\right]=\left(X_{2}+\frac{4}{3} I X_{4}\right),} \\
{\left[X_{4}, X_{5}\right]=\left(X_{3}-I Y_{2}\right),\left[X_{5}, Y_{2}\right]=X_{4} .}
\end{gathered}
$$

The parameter $I$ is a semi-invariant and $I^{2}$ is a bona-fide invariant (Cartan [C] writes that $I$ is obtained from the forth root, so some power of it is an invariant; we shall see that this power is 2).

The above description of $\mathfrak{g}$ is however not convenient, since the restriction of $\operatorname{ad}\left(X_{5}\right)$ to the derived algebra $\mathfrak{g}_{2}=\left\langle X_{1}, X_{2}, X_{3}, X_{4}, Y_{2}\right\rangle$ is not normalized $\left(\operatorname{ad}\left(Y_{1}\right)\right.$ acts like the grading element). This operator is semi-simple and diagonalizing it (changing the basis in $\left\langle X_{2}, X_{3}, X_{4}, Y_{2}\right\rangle$ ) is equivalent to passing to the basis $\left\{W_{i}\right\}_{i=1}^{7}$.

In this new basis the description of $\mathfrak{g}$ is the following. The derived series is $\mathfrak{g}_{2}=\left\langle W_{1}, W_{2}, W_{3}, W_{5}, W_{7}\right\rangle, \mathfrak{g}_{3}=\left\langle W_{3}\right\rangle$, and $\mathfrak{g}_{2}$ is the Heisenberg algebra $\mathfrak{h}=\mathfrak{h}_{-1} \oplus \mathfrak{h}_{-2}$ (now indices denote the grading) given by the symplectic form on $\mathfrak{h}_{-1}=\left\langle W_{1}, W_{2}, W_{5}, W_{7}\right\rangle$ with values in $\mathfrak{h}_{-2}=\left\langle W_{3}\right\rangle$. In other words, the non-trivial brackets are

$$
\left[W_{1}, W_{5}\right]=W_{3},\left[W_{2}, W_{7}\right]=\frac{-1}{m} W_{3}
$$

(we keep the normalizing factor).

This is extended to $\tilde{\mathfrak{h}}=\mathfrak{h} \oplus \mathbb{R} \cdot W_{4}$ by the grading element $\left.\operatorname{ad}\left(W_{4}\right)\right|_{\mathfrak{h}_{k}}=$ $k \cdot$ id. Finally $\mathfrak{g}$ is obtained from $\tilde{\mathfrak{h}}$ by right extension via the element in $H^{1}(\tilde{\mathfrak{h}}, \tilde{\mathfrak{h}})$ given as follows $(m \neq 1 / 2)$ :

$$
-\operatorname{ad}\left(W_{6}\right)=m W_{2} \otimes \theta_{2}+W_{3} \otimes \theta_{3}+W_{5} \otimes \theta_{5}+(1-m) W_{7} \otimes \theta_{7}
$$

$\left(\theta_{i}\right.$ is the coframe dual to $\left.W_{i}\right)$.

Subtracting the trace we get the operator $A=\operatorname{ad}\left(W_{6}-\frac{1}{2} W_{4}\right)$ on $\mathfrak{h}_{-1}$ (it vanishes on $\mathfrak{h}_{-2}$ ), whose conformal class is an invariant of the Lie algebra $\mathfrak{g}$. 
From the spectrum $\operatorname{Sp}(A)=\left\{ \pm \frac{1}{2}, \pm\left(\frac{1}{2}-m\right)\right\}$ we obtain the absolute invariant $\lambda=\operatorname{Tr}\left(A^{4}\right) / \operatorname{Tr}\left(A^{2}\right)^{2}$. Passing to $2(1-2 \lambda)$ we get the invariant

$$
J=\frac{(1-2 m)^{2}}{\left(1-2 m+2 m^{2}\right)^{2}} .
$$

Calculating the invariant $J$ in the Cartan basis (using $\operatorname{ad}\left(X_{5}\right)$ ) we get $J=\frac{9}{25}\left(1+I^{-2}\right)$. Now we can relate the parameters:

$$
I= \pm \frac{i\left(1-2 m+2 m^{2}\right)}{2 \sqrt{(m+1)(m-1 / 3)(m-2 / 3)(m-2)}} .
$$

We see that poles of $I$ correspond to maximal finite-dimensional symmetry algebra $G_{2}$, while linearizable cases correspond to $I= \pm \frac{3}{4}$.

For the exceptional case $m=1 / 2$ the spectrum is multiple (this happens also for $m=0,1$ ), and this is the only case, when $A$ is not semi-simple:

$$
-\operatorname{ad}\left(W_{6}-\frac{1}{2} W_{4}\right)=\frac{1}{2} W_{5} \otimes \theta_{5}-\frac{1}{2} W_{1} \otimes \theta_{1}+\frac{1}{2} W_{2} \otimes \theta_{7} .
$$

It is more convenient to describe the equivalence classes in terms of the parameter $k=2 m-1$. On the real line $\mathbb{R}^{1}(k)$ we have the action of the group $\mathbb{Z}_{2} \oplus \mathbb{Z}_{2}$ with generators $k \mapsto-k, k \mapsto k^{-1}$ (the latter is not defined at 0 ). Its orbits correspond to the equivalent Monge equations (5). In particular, the cases of symmetry dimensions $\infty$ and 14 corresponds to the orbits of $k=1$ and $k=3$, and the exceptional case of Jordan block corresponds to $k=0$. The orbit space is the union of two $k$-intervals $[0,1 / 3] \cup[1 / 3,1]$. The invariant $I$ expresses via $k$ so:

$$
I^{2}=\frac{\left(k^{2}+1\right)^{2}}{\left(k^{2}-9\right)\left(1 / 9-k^{2}\right)} .
$$

Consider the next case of rank 2 distributions in $\mathbb{R}^{6}$, which are not reducible to the previous case of $(2,5)$-distributions. Then [DZ, AK] its symmetry algebra is at most 11-dimensional. It is enough to restrict to the following Monge equations in this case (in dimension 6 not all rank 2 distributions are realized as this underdetermined ODE, the other two cases correspond to hyperbolic structures involving only second derivatives and the elliptic structures that can be realized only as Monge systems [AK] but their symmetry algebras are at most 8dimensional)

$$
y^{\prime}=F\left(x, y, z, z^{\prime}, z^{\prime \prime}, z^{\prime \prime \prime}\right) .
$$

Non-degeneracy condition $F_{z_{3} z_{3}} \neq 0$ is sufficient for finite-dimensionality. Here the maximal symmetric model is unique and is given by

$$
y^{\prime}=\left(z^{\prime \prime \prime}\right)^{2} .
$$


We refer to $\mathrm{AK}$ for the description of the symmetry algebra (both as internal and as external higher symmetries).

The submaximal case is given by the family $(\epsilon \in \mathbb{C})$

$$
y^{\prime}=\left(z^{\prime \prime \prime}\right)^{2}+\epsilon^{2}\left(z^{\prime \prime}\right)^{2} .
$$

The symmetry algebra is 9-dimensional and is represented as follows:

$$
\begin{gathered}
\operatorname{sym}\{(7)\}=\left\langle W_{1}=\partial_{x}, W_{2}=\partial_{y}, W_{3}=\partial_{z},\right. \\
W_{4}=2 y \partial_{y}+z \partial_{z}+z_{1} \partial_{z_{1}}+z_{2} \partial_{z_{2}}+z_{3} \partial_{z_{3}}, W_{5}=x \partial_{z}+\partial_{z_{1}}, \\
W_{6}=2 \epsilon^{2} z_{1} \partial_{y}+\frac{1}{2} x^{2} \partial_{z}+x \partial_{z_{1}}+\partial_{z_{2}}, \\
W_{7}=2\left(z_{2}+\epsilon^{2}\left(x z_{1}-z\right)\right) \partial_{y}+\frac{1}{6} x^{3} \partial_{z}+\frac{1}{2} x^{2} \partial_{z_{1}}+x \partial_{z_{2}}+\partial_{z_{3}}, \\
W_{8}=e^{-\epsilon x}\left(2 \epsilon^{3} z_{2} \partial_{y}-\partial_{z}+\epsilon \partial_{z_{1}}-\epsilon^{2} \partial_{z_{2}}+\epsilon^{3} \partial_{z_{3}}\right), \\
\left.W_{9}=e^{\epsilon x}\left(2 \epsilon^{3} z_{2} \partial_{y}+\partial_{z}+\epsilon \partial_{z_{1}}+\epsilon^{2} \partial_{z_{2}}+\epsilon^{3} \partial_{z_{3}}\right)\right\rangle .
\end{gathered}
$$

Its Lie algebra structure is the following: the derived series of $\mathfrak{g}$ is $\mathfrak{g}_{2}=$ $\left\langle W_{2}, W_{3}, W_{5}, W_{6}, W_{7}, W_{8}, W_{9}\right\rangle, \mathfrak{g}_{3}=\left\langle W_{2}\right\rangle$, where $\mathfrak{g}_{2}$ is the Heisenberg algebra again $\mathfrak{h}=\mathfrak{h}_{1} \oplus \mathfrak{h}_{2}$ (the indices denote the grading) given by the symplectic form on $\mathfrak{h}_{1}=\left\langle W_{3}, W_{5}, W_{6}, W_{7}, W_{8}, W_{9}\right\rangle$ with values in $\mathfrak{h}_{2}=\left\langle W_{2}\right\rangle$ :

$$
\begin{gathered}
{\left[W_{3}, W_{7}\right]=-2 \epsilon^{2} W_{2},\left[W_{5}, W_{6}\right]=2 \epsilon^{2} W_{2},} \\
{\left[W_{6}, W_{7}\right]=2 W_{2},\left[W_{8}, W_{9}\right]=-4 \epsilon^{5} W_{2}}
\end{gathered}
$$

(normal form is achieved by the shift of $\left(W_{6}, W_{7}\right)$ by $\frac{1}{2 \epsilon^{2}}\left(W_{3}, W_{5}\right)$ ). Then the 2-dimensional right extension from $\mathfrak{h}$ to $\mathfrak{g}$ is achieved via grading element $W_{4},\left.\operatorname{ad}\left(W_{4}\right)\right|_{\mathfrak{h}_{k}}=k \cdot \mathrm{id}$, and the derivation

$$
\mathrm{W}_{1}=W_{3} \otimes \theta_{5}+W_{5} \otimes \theta_{6}+W_{6} \otimes \theta_{7}-\epsilon W_{8} \otimes \theta_{8}+\epsilon W_{9} \otimes \theta_{9} .
$$

Let us now demonstrate the sub-maximal property.

Theorem 7. There exists no rank 2 distribution in $\mathbb{R}^{6}$ with symbol of general position (i.e. growth vector being $(2,3,5,6)$ ) that possesses a 10-dimensional symmetry algebra.

To demonstrate this we will use Corollary 6 .

Proof. The maximal symmetry algebra, corresponding to (6) , was calculated in $\mathrm{AK}]$ : the graded Lie structure is

$$
\begin{aligned}
& \mathfrak{g}=\mathfrak{g}_{-4} \oplus \mathfrak{g}_{-3} \oplus \mathfrak{g}_{-2} \oplus \mathfrak{g}_{-1} \oplus \mathfrak{g}_{0} \oplus \mathfrak{g}_{1} \\
&=\left\langle Z_{0}\right\rangle \oplus\left\langle Y_{0}, Z_{1}\right\rangle \oplus\left\langle Z_{2}\right\rangle \oplus\left\langle S_{0}, Z_{3}\right\rangle \oplus\left\langle S_{1}, R, Z_{4}\right\rangle \oplus\left\langle S_{2}, Z_{5}\right\rangle
\end{aligned}
$$


and the commutators are (we list only non-trivial ones)

$$
\begin{gathered}
{\left[S_{0}, S_{1}\right]=S_{0},\left[S_{0}, S_{2}\right]=2 S_{1},\left[S_{1}, S_{2}\right]=S_{2},} \\
{\left[S_{0}, Z_{i}\right]=Z_{i-1},\left[S_{1}, Z_{i}\right]=\left(i-\frac{5}{2}\right) Z_{i},\left[S_{2}, Z_{i}\right]=(i+1)(i-5) Z_{i+1},} \\
{\left[Z_{0}, Z_{5}\right]=2 Y_{0},\left[Z_{1}, Z_{4}\right]=-2 Y_{0},\left[Z_{2}, Z_{3}\right]=2 Y_{0}} \\
{\left[Y_{0}, R\right]=Y_{0},\left[Z_{i}, R\right]=\frac{1}{2} Z_{i} .}
\end{gathered}
$$

Its Levi decomposition is

$$
\mathfrak{g}=\mathrm{sl}_{2}+\mathfrak{m}^{8}=\left\langle S_{i}\right\rangle \oplus\left\langle R, Y_{0}, Z_{j}\right\rangle .
$$

It follows that any 10-dimensional graded subalgebra shall contain the 8-dimensional space $\left\langle S_{1}, Y_{0}, Z_{j}\right\rangle$, to which a 2-dimensional subspace in $\left\langle S_{0}, S_{2}, R\right\rangle$ shall be added.

This can be reduced to removing from $\mathfrak{g}$ either $R$ or $S_{2}$ (in fact, internally any subalgebra of $\mathrm{sl}_{2}$ is conjugated to $\left\langle S_{0}, S_{1}\right\rangle$, but in our case the semi-simple part enters with the graded representation, and so there are more cases; however e.g. removal of $S_{0}$ is much simpler and we restrict to the most complicated cases). This is a 10-dimensional subalgebra $\mathfrak{h} \subset \mathfrak{g}$, but we need to check if it has a deformation in the spirit of Corollary 6 .

The first choice $\mathfrak{h}=\left\langle S_{i}, Y_{0}, Z_{j}\right\rangle$ is easy: The $\mathrm{sl}_{2}$ subalgebra is rigid upon deformation $\mathfrak{h} \rightsquigarrow h$, as well as its module $\mathfrak{m}^{7}=\left\langle Y_{0}, Z_{j}\right\rangle$. The Lie algebra structure on $h=\mathrm{sl}_{2}+\mathfrak{m}^{7}$ is given by a choice of $\mathrm{sl}_{2}$-equivariant morphism $\Lambda^{2} \mathfrak{m}^{7} \rightarrow h$.

Denoting by $V$ the tautological (2D) representation and by $\varepsilon$ the trivial one, we have $\mathfrak{m}^{7}=S^{5} V \oplus \varepsilon$ and consequently $\Lambda^{2} \mathfrak{m}^{7}=S^{8} V \oplus$ $S^{5} V \oplus S^{4} V \oplus \varepsilon$. Notice that the adjoint representation, equivalent to $S^{2} V$ is not among the summands. Thus by Schur's lemma the only morphism is the projection to $S^{5} V \oplus \varepsilon$ (given by two coefficients).

Therefore the only possible Lie algebra structure is given by the commutators $\left[Z_{i}, Z_{j}\right]=\lambda_{i j} Y_{0}$ and $\left[Z_{i}, Y_{0}\right]=c Z_{i}$ (the brackets with $\mathrm{sl}_{2}$ are the same as in $\left.\mathfrak{g}\right)$. Acting on the first equation by $\operatorname{ad}\left(S_{1}\right)$ we obtain $\lambda_{i j}=0$ unless $i+j=5$, and acting on it by $\operatorname{ad}\left(S_{0}\right)$ we get $\lambda_{0,5}=-\lambda_{1,4}=\lambda_{2,3}$ and this can be normalized to 2 (it is non-zero by the deformation constraints). Now the Jacobi identity for the triple $\left(Z_{i}, Z_{5-i}, Z_{k}\right)$ yields $c=0$ and we recover the structure of $\mathfrak{h}$. So no non-trivial deformation is possible

The second choice $\mathfrak{h}=\left\langle S_{0}, S_{1}, Y_{0}, Z_{j}, R\right\rangle$ is more complicated because the semisimple part is gone. Let us start by calculating the usual deformation. The operator $\operatorname{ad}\left(S_{1}\right)$ has spectrum $\left\{-1,0,0,0, \pm \frac{5}{2}, \pm \frac{3}{2}, \pm \frac{1}{2}\right\}$. The simple eigenvalues will be perturbed and we can normalize -1 to be fixed. This restores the solvable subalgebra $\left\langle S_{0}, S_{1}\right\rangle$.

Next the Jacobi identity yields $\left[S_{1},\left[S_{0}, Z_{i}\right]\right]=\left(\lambda_{i}-1\right)\left[S_{0}, Z_{i}\right]$ provided $\left[S_{1}, Z_{i}\right]=\lambda_{i} Z_{i}$. This implies $\left[S_{0}, Z_{i}\right]=Z_{i-1}$ and $\left[S_{1}, Z_{i}\right]=\left(i-\frac{5}{2}+\lambda\right) Z_{i}$ 
for some $\lambda$. We define $Y_{0}=\frac{1}{2}\left[Z_{0}, Z_{5}\right]$ and then calculate if $\lambda \neq 0$ but small: $\left[S_{0}, Y_{0}\right]=0,\left[S_{1}, Y_{0}\right]=2 \lambda Y_{0}$. We can proceed and get in this way a non-trivial deformation, but it will not be of the type described in Corollary 6.

So let us use the assumption on filtration, namely that after passing to grading we have a monomorphism $\mathfrak{h} \rightarrow \mathfrak{g}$ (the filtration in $\mathfrak{g}$ is $\left.F_{i}=\oplus_{k \geq i} \mathfrak{g}_{k}\right)$. This yields $\lambda=0$, and then it is not difficult to deduce that the brackets for $\left[Z_{i}, Z_{j}\right]$ are the same as in $\mathfrak{g}$.

Indeed, since $\operatorname{ad}\left(S_{1}\right)\left[Z_{i}, Z_{j}\right]=(i+j-5)\left[Z_{i}, Z_{j}\right]$ the latter commutator can be non-zero only for $i+j=4$ or 5 . Applying $\operatorname{ad}\left(S_{0}\right)$ to $\left[Z_{i+1}, Z_{j}\right]=0$ with $i+j=5$ we get $\left[Z_{i}, Z_{j}\right]=-\left[Z_{i+1}, Z_{j-1}\right]$, from where $\left[Z_{i}, Z_{5-i}\right]=(-1)^{i} 2 Y_{0}$. Applying ad $\left(S_{0}\right)$ to this starting from $i=0$ we get $\left[Z_{i}, Z_{4-i}\right]=0$.

Now by Leibniz rule it follows that $\left[S_{i}, Y_{0}\right]=0$, and so we restore all commutators relations from $\mathfrak{g}$ for the subalgebra $\left\langle S_{0}, S_{1}, Y_{0}, Z_{j}\right\rangle$.

It remains to add $R$. By filtration reason the following relations should hold for some constant coefficients:

$$
\left[S_{0}, R\right]=\alpha Z_{5}+\beta S_{1}+\gamma R+\delta Z_{4}, \quad\left[S_{1}, R\right]=\sigma Z_{5}, \quad\left[Z_{5}, R\right]=0 .
$$

Applying to the last equality $\operatorname{ad}\left(S_{0}\right)$ several times (and using the first equality) we successively get formulae for $\left[Z_{i}, R\right]$, which we do not reproduce all. When $i=-1\left(Z_{-1}=0\right)$ we obtain

$$
\begin{aligned}
-3 \gamma Z_{0}- & \frac{5}{2} \gamma(7 \beta-3 \gamma) Z_{1}+5 \gamma^{2}(7 \beta-\gamma) Z_{2}-\frac{3}{2} \gamma^{3}(21 \beta-5 \gamma) \\
& +\gamma^{4}(14 \beta-3 \gamma) Z_{4}-\frac{1}{2} \gamma^{5}(5 \beta-\gamma) Z_{5}-2(\alpha+\delta \gamma) Y_{0}=0,
\end{aligned}
$$

which implies $\alpha=\gamma=0$.

Now applying ad $\left(S_{0}\right)$ to the middle equality in (8) we obtain $\beta S_{1}+$ $\frac{5}{2} \delta Z_{4}=\sigma Z_{4}$, so that $\beta=0, \sigma=\frac{5}{2} \delta$. The last number can be nonzero, but it is removed by the change $R \mapsto R-\frac{2}{5} \sigma Z_{5}$. This restores all the commutators and so we get $h=\mathfrak{h} \subset \mathfrak{g}$. Thus no non-trivial deformation exists.

Thus we see that the rank 2 distribution in $\mathbb{R}^{6}$ corresponding to (17) actually represents a sub-maximal case. The structure of maximal symmetry is unique according to $\mathrm{AK}$, DZ, but this is not so for the sub-maximal case; here are some other models representing rank 2 distributions in $\mathbb{R}^{6}$ with 9-dimensional symmetry algebra:

$$
y^{\prime}=\left(z^{\prime \prime \prime}\right)^{2}+\epsilon^{2}\left(z^{\prime}\right)^{2} \quad \text { and also } \quad y^{\prime}=\left(z^{\prime \prime \prime}\right)^{2}+\epsilon^{2} z^{2} .
$$

Another interesting series of symmetric models corresponding to rank 2 distributions in $\mathbb{R}^{6}$ is

$$
y^{\prime}=\left(z^{\prime \prime \prime}\right)^{m} .
$$


For generic $m$ the symmetry algebra is 7-dimensional, but for some values like $\frac{1}{2}$ and $\frac{1}{3}$ it is 8 -dimensional, and these special systems together with elliptic and hyperbolic Monge equations from [AK] yield sub-submaximal symmetric models of 2-distributions in $\mathbb{R}^{6}$.

In higher dimensions the maximally symmetric Monge equations are given by

$$
y^{\prime}=\left(z^{(n)}\right)^{2} .
$$

They represent rank 2 distributions in $\mathbb{R}^{n+3}$ with the symmetry algebra of dimension $2 n+5$. The sub-maximal case is realized by any of the equations

$$
y^{\prime}=\left(z^{(n)}\right)^{2}+\epsilon\left(z^{(j)}\right)^{2}
$$

with $0 \leq j<n$; its symmetry algebra is $(2 n+3)$-dimensional. Thus in this case the gap is 2 .

For other equations, like elliptic and hyperbolic Monge equations [AK], the gap takes the minimal possible value 1 .

\section{Conclusion}

We have observed that the gap phenomenon exists (i.e. the lacune exceeds 1 ) if the maximal symmetry algebra has a semi-simple part; the larger is the dimension of the latter the larger is expected the gap.

For geometric structures associated to distributions (in which case the maximal symmetry algebra is graded and is given by the Tanaka theory) we have been able to explain the phenomenon and presented a tool to perceive the gap.

Also in the latter case the maximal symmetry model (as well as its algebra) is unique, though it is not true in general (e.g. for Riemannian metric with maximal amount of Killing fields). Sub-symmetric models are seldom unique.

Remark also that maximal symmetry algebras act transitively on the manifold where the structure lives (in other words the maximal models are homogeneous). This still holds for the sub-maximal models related to distributions, e.g. in the discussed cases (5) and (7). In general sub-maximal situation even this is not always true (compare again the case of local Killing fields on surfaces). In the lower-dimensional case like sub-submaximal (9), and also for Cartan's involutive systems with 6-dimensional algebras [C, p.170], this property fails.

Understanding of maximal and sub-maximal symmetric models in general still remains an interesting open problem.

\section{REFERENCES}

[AK] I. M. Anderson, B. Kruglikov, Rank 2 distributions of Monge equations: symmetries, equivalences, extensions, Adv. Math. 228, issue 3, 1435-1465 (2011). 
[BM] M. Bialy, A. Mironov, Rich quasi-linear system for integrable geodesic flows on 2-torus, Discrete Contin. Dyn. Syst. 29, no. 1, 81-90 (2011).

[C] E. Cartan, Les systèmes de Pfaff, à cinq variables et les équations aux dérivées partielles du second ordre, Ann. Sci. École Norm. Sup. (3) 27, 109-192 (1910).

[D] G. Darboux, Leçons sur la théorie générale des surfaces et les applications géométriques du calcul infinitésimal, II partie (1887), IV partie (1896). Gauthier-Villar, Paris.

[DZ] B. Doubrov, I. Zelenko, On local geometry of nonholonomic rank 2 distributions, J. London Math. Society, 80, 545-566 (2009).

[Du] M. Dunajski, S. West, Anti-Self-Dual Conformal Structures in Neutral Signature, arXiv:math/0610280 in 'Recent developments in pseudoRiemannian Geometry', ESI Lectures in Mathematics and Physics (2008), edited by D. Alekseevsky and H. Baum.

$\left[\mathrm{E}_{1}\right] \quad$ I. P. Egorov, Riemannian spaces of the first three lacunary types in the geometric sense, Dokl. Akad. Nauk SSSR 150, 730-732 (1963).

$\left[\mathrm{E}_{2}\right]$ I. P. Egorov, Motions in the spaces with affine connection, 2nd ed. (Russian), URSS (2009).

[Ei] L. P. Eisenhart, Riemannian Geometry, Princeton University Press (1949).

[G] E. Goursat, Lecons sur l'intégration des équations aux dérivées partielles du second ordere, II, Hermann, Paris (1898)

[Ki] K. Kiyohara, Compact Liouville surfaces, J. Math. Soc. Japan 43, no. 3, 555-591 (1991).

[Ko] S. Kobayashi, Transformation Groups in Differential Geometry, SpringerVerlag, Berlin (1972).

[Kol] V. Kolokol'tsov, Polynomial integrals of geodesic flows on compact surfaces (Russian), PhD thesis, Moscow State University (1984).

$\left[\mathrm{K}_{1}\right] \quad$ B. Kruglikov, Invariant characterization of Liouville metrics and polynomial integrals, J. Geom. Phys. 58, no. 8, 979-995 (2008).

$\left[\mathrm{K}_{2}\right]$ B. Kruglikov, Point classification of second order ODEs: Tresse classification revisited and beyond (with an appendix by B. Kruglikov and V. Lychagin), in: Abel Symp. 5, Differential equations: geometry, symmetries and integrability, 199-221, Springer, Berlin (2009).

$\left[\mathrm{K}_{3}\right]$ B. Kruglikov, Finite-dimensionality in Tanaka theory, Ann. Inst. H. Poincaré Anal. Non Linéaire 28, no. 1, 75-90 (2011).

$\left[\mathrm{K}_{4}\right] \quad$ B. Kruglikov, Symmetries of almost complex structures and pseudoholomorphic foliations, arXiv:1103.4404 (2011).

[KL] B. Kruglikov, V. Lychagin, Geometry of Differential equations, In: Handbook on Global Analysis, D.Krupka and D.Saunders Eds., 725-771, 1214, Elsevier Sci. (2008).

[KLV] I. S. Krasilschik, V. V. Lychagin, A. M. Vinogradov, Geometry of jet spaces and differential equations, Gordon and Breach (1986).

$\left[\mathrm{KM}^{2} \mathrm{~S}\right]$ V. Kiosak, V. Matveev, I. Mikesh, I. Shandra, On the degree of geodesic mobility of Riemannian metrics, Math. Notes 87, no. 4, 628-629; Engl. transl. pp: 586-587 (2010).

[MS] V. Matveev, V. Shevchishin, Two-dimensional superintegrable metrics with one linear and one cubic integral, J. Geom. Phys. 61, no. 8, 1353-1377 (2011).

[MT] V. Matveev, P. Topalov, Trajectory equivalence and corresponding integrals, Regular and Chaotic Dynamics 3, no. 2, 30-45 (1998). 
[P] V. Patrangenaru, Lorentz manifolds with the three largest degrees of symmetry, Geom. Dedicata 102, 25-33 (2003).

[T] N. Tanaka, On differential systems, graded Lie algebras and pseudo-groups, J. Math. Kioto Univ. 10, no.1, 1-82 (1970).

[Tr] A. Tresse, Détermination des invariants ponctuels de l'équation différentielle ordinaire de second ordre $y^{\prime \prime}=\omega\left(x, y, y^{\prime}\right)$, Mémoire couronné par l'Académie Jablonowski; S. Hirkel, Leipzig (1896).

[YK] K. Yano, M. Kon, Structures on manifolds, World Scientific Publishing (1984).

[Z] R. Zimmer, On the automorphism group of a compact Lorentz manifold and other geometric manifolds, Invent. Math. 83, no. 3, 411-424 (1986).

Department of Mathematics and Statistics, University of Troms $\varnothing$, Troms $\varnothing$ 90-37, Norway._E-MAIL: Boris.Kruglikov@Uit.no. 